[Bull. Agr. Chem. Soc. Japan, Vol. 22, No. 1, p. 57 58, 1958]

\title{
Isolation of $\gamma$-Phytadiene from the Essential Oil of Tobacco Leaf
}

Sir :

We have already reported ${ }^{11}$ the presence of an unsaturated aliphatic hydrocarbon resembling myrcene, having a conjugated doublebond in its molecule, contained in the neutral fraction of the essential oil of the aged Japanese Flue-cured tobacco leaf. Further studies were conducted on the structure of this compound which was also separated from the hydrocarbon fraction $(\mathrm{H}-1)^{2)}$ of the aged Japanese Burley tobacco leaf. Molecular weight determination proved that this compound belongs to diterpenes and catalytic hydrogenation indicated that it has two double-bonds in its molecule. Upon decomposition of its ozonide by water, formaldehyde and a monobasic higher fatty acid were obtained. In addition to these results, according to the formation of an aduct with $\alpha$-naphthoquinone and infrared spectrophotometric investigations of a hydrocarbon separated from the commercially obtained phytol, this compound was estimated as a new hexahydroditerpene $(4,8,12$-trimethyltridecanyl-(1)-2-butadiene-1,3), namely, (7,11,15trimethyl-3-methylene-hexadecene-1), $\gamma$-phytadiene.
Physical and chemical constants of $\gamma$-phytadiene:

b.p. $144-146^{\circ} / 3.5 \mathrm{~mm}: d_{20}^{20} 0.8168: n_{\mathrm{D}}^{20} 1.4628$ : $[\alpha]_{\mathrm{D}}^{20}+0.03$ : M.R. 93.88: M.W. Found by cryoscopic method 269; Galcd. for $\mathrm{C}_{20} \mathrm{H}_{38}$ 278: Anal. Found: C, 86.55; H, 13.25. Calcd. for $\mathrm{C}_{20} \mathrm{H}_{38}$ : C, 86.24; H, 13.75 .

Infrared spectrum of $\gamma$-phytadiene (Fig. 1) indicated that this compound has two unsaturated radicals which was affirmed to conjugate as $\mathrm{CH}_{2}=\mathrm{CR}-\mathrm{CH}=\mathrm{CH}_{2}$. Ultraviolet spectrum of $\gamma$-phytadiene indicated a maximum absorption at $224 \mathrm{~m} \mu, \varepsilon=15475$, and those of myrcene are reported as $\left.224.5 \mathrm{~m} \mu, \varepsilon=15350^{3}\right)$ and $17030^{4)}$. As in the case of infrared spectra, the presence of the conjugated double-bond was also identified by the ultraviolet spectrum.

Ozonization was performed in the chloroform solution and the resulted ozonide was decomposed with water. Formaldehyde (2,4dinitrophenylhydrazone, m.p. $\left.156^{\circ}\right)$, and a higher aliphatic acid ( $p$-phenylphenacyl ester, m.p. $52.5-53.1^{\circ}$ ), were obtained from the decomposed product of ozonide. Elementary analysis of this higher aliphatic acid resulted as $\mathrm{C}_{16}$ or $\mathrm{C}_{17}$. The infrared spectrum of $p$ phenylphenacyl ester of this acid is shown

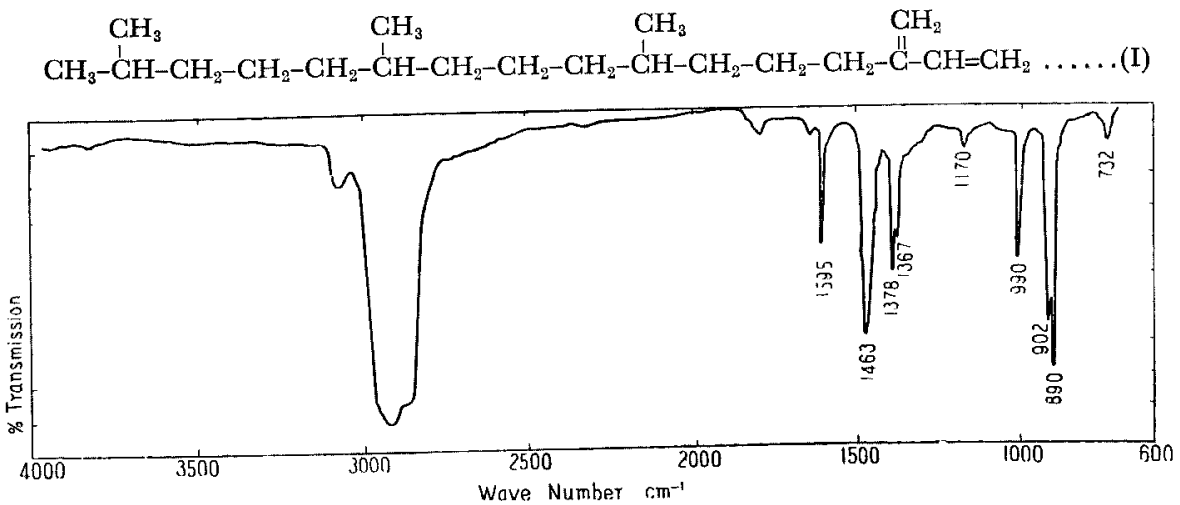

FIG. $\overline{1}$. Infrared Spectrum of $r$-Phytadiene.

1) I. Ōnishi, H. Tomita and T. Fukuzumi, This Bulletin, 20, $61(1956)$.

2) I. Ōnishi, M. Nagasawa, H. Tomita and T. Fukuzumi. This Bulletin, (received).
3) R.D. Walker and J.E. Hawkins, J. Am. Chem. Soc., 74. 4209 (1952)

4) R.T. O'Connor and L.A. Goldblatt, Anal. Chem, 26, 1726 (1954). 


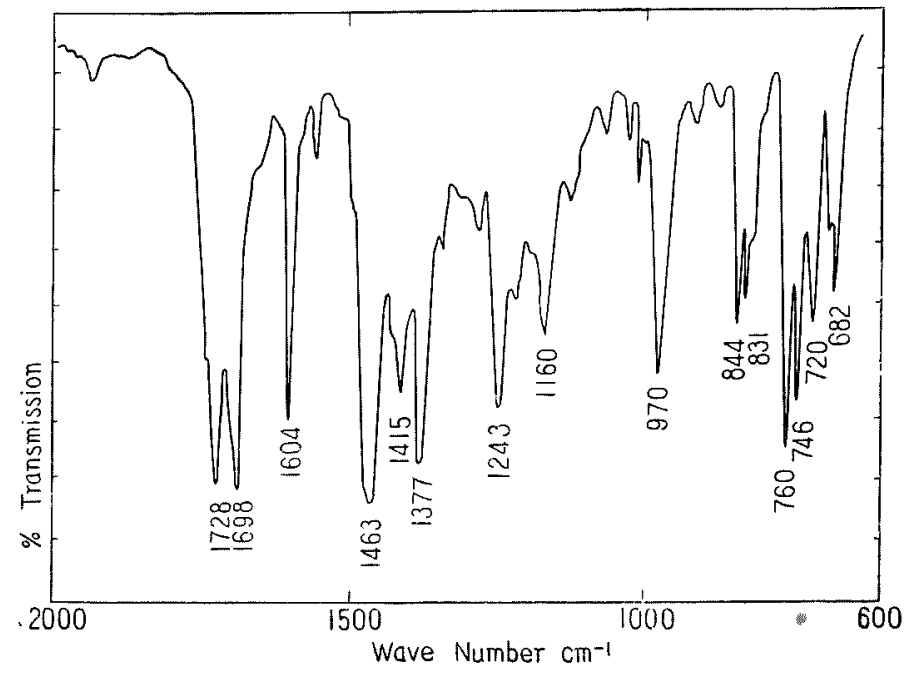

FIG. 2. Infrared Spectrum of $p$-Phenylphenacyl ester of an Acid Obtained by Water Decomposition of $\gamma$-Phytadiene-Ozonide (in nujol).

in Fig. 2.

$\alpha$-Naphthoquinone was reacted with $\gamma$-phytadiene and a substance, containing yellow crystals thus obtained, was separated by a liquid chromatography. A white crystal, m.p. $34.5-34.8^{\circ}$, was obtained from the eluate of a yellow band, which was eluted prior to that of the unreacted reagent. Anal. Found: C, 82.29; H, 10.06. Calcd. for $\mathrm{C}_{30} \mathrm{H}_{14} \mathrm{O}_{2}: \mathrm{C}, 82.5 \mathrm{I}$; $\mathrm{H}, 10.16$. Infrared spectrum of this aduct is shown in Fig. 3.

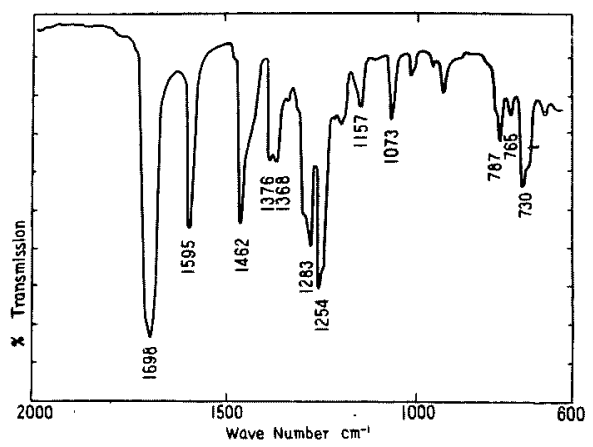

FIG. 3. Infrared Spectrum of an Aduct of $\gamma$ Phytadiene with $\alpha$-Naphthoquinone.

Judging from the infrared spectrum of this compound which indicated the presence of the isopropyl radical at the end of its molecule, and also from other physical properties suggesting that this compound belongs to diterpenes, this compound was supposed to be an isomer of phytadieness), derived by the dehydrogenation of phytol. The infrared spectrum of a hydrocarbon, separated from the commercially obtained phytol in the purification process, agreed well with that of the above-mentioned newly isolated $\gamma$-phytadiene. From these experimental results, the molecular structure of $\gamma$-phytadiene (I) was supposed to be reasonable.

The aged Japanese Burley tobacco leaf contains more amount of $\gamma$-phytadiene $(56.45$ $\mathrm{mg} / \mathrm{kg}$ of leaf) than the aged Japanese Fluecured leaf (20.06 mg/kg of leaf).

\section{Isao ŌNIsHI \\ Masatoshi Nagasawa Hideo Tomita Tetsuo Fukuzumi}

Tobacco Research Department, Central Research Institute, Japan Monopoly Corporation.

Received October 10, 195\% 5) P. Karrer, A. Kugler and H. Simon, He'v, Chim, Acka.,
27, 1006 (1944). 